\title{
Cartographier les réseaux d'une philosophie franco-italienne des techniques
}

Compte rendu de deux ouvrages collectifs

\section{Cléo Collomb}

\section{(2) OpenEdition}

\section{Journals}

\section{Édition électronique}

URL : https://journals.openedition.org/artefact/10549

DOI : $10.4000 /$ artefact.10549

ISSN : 2606-9245

\section{Éditeur :}

Association Artefact. Techniques histoire et sciences humaines, Presses universitaires du Midi

\section{Édition imprimée}

Date de publication : 7 octobre 2021

Pagination : 467-475

ISBN : 978-2-8107-0755-3

ISSN : 2273-0753

\section{Référence électronique}

Cléo Collomb, «Cartographier les réseaux d'une philosophie franco-italienne des techniques », Artefact [En ligne], 14 | 2021, mis en ligne le 07 octobre 2021, consulté le 07 octobre 2021. URL : http:// journals.openedition.org/artefact/10549; DOI : https://doi.org/10.4000/artefact.10549

\section{(@) $\odot \Theta \Theta$}

Artefact, Techniques, histoire et sciences humaines est mise à disposition selon les termes de la Licence Creative Commons Attribution - Pas d'Utilisation Commerciale - Pas de Modification 4.0 International. 


\section{Cartographier les réseaux d'une philosophie franco-italienne des techniques}

\section{Compte rendu de deux ouvrages collectifs}

\section{Cléo Collomb}

T 'objectif de ces deux ouvrages collectifs est similaire : donner à voir l'existence de véritables communautés de philosophes des techniques en France et en Italie. Le geste n'est évidemment pas neutre: en donnant à voir, les livres dont il est question participent à la constitution de ces communautés, ils les font exister. Cette mise en visibilité instituante est peut-être d'autant plus percutante qu'elle traverse les langues. Les philosophes italiens sont publiés en français, les philosophes français, en anglais. Le lecteur reçoit donc à chaque fois des pensées philosophiques en partie préparées pour l'extérieur, pour l'en-dehors. Sans existence préalable, ces philosophies des techniques - française et italienne - n'auraient pas pu traverser les frontières, mais c'est aussi en les traversant qu'elles sont instaurées et se consolident. French Philosophy of Technology et Perspectives italiennes contemporaines en philosophie des techniques sont des livres performatifs. Et c'est en partie ce qui les rend captivants, en particulier pour le lecteur francophone - même celui dont l'anglais s'avérerait tâtonnant : il y a quelque chose de joyeux dans le fait de découvrir sa propre communauté, mais depuis une situation de réception décentrée, dans le fait de redécouvrir l'ici depuis le là-bas, le nous depuis le eux. Une véritable expérience qui nous rappelle à quel point être lecteur est un acte intéressant. Mais c'est

99 Cléo Collomb, « Cartographier les réseaux d’une philosophie franco-italienne des techniques. Compte rendu de deux ouvrages collectifs », Artefact, 14, 2021, p. 467475. 
aussi une expérience qui interpelle : faut-il qu'il y ait un eux pour qu'il y ait un nous? Faut-il que je sois eux pour me frotter à l'existence du nous ?

Les chapitres de présentation des deux livres expliquent bien à quel point la philosophie des techniques bénéficie d'une reconnaissance et d'une institutionnalisation aux Pays-Bas et dans le monde anglo-saxon, contrairement à ce qu'il se passe en France et en Italie. Il s'agit donc bien, dans les cas français et italien, d'un manque de légitimité et de visibilité et non pas d'un défaut d'existence. Dans les chapitres de présentation toujours, les lecteurs trouveront une synthèse très claire des spécificités des philosophies italienne et française des techniques. Premièrement, elles partagent toutes deux un intérêt pour la matérialité concrète des techniques. Tout comme leurs cousines américaines et néerlandaises, centrées sur les artéfacts (Verbeek ; Kroes et Meijers) ou les médiations (Ihde) techniques, elles ont elles aussi connu leur "tournant empirique " - en témoignent l'intérêt pour le concept simondonien d' " objet technique " et les différents travaux attachés à des objets concrets comme les plastiques, les jeux vidéos, les nano-objets, les instruments scientifiques, etc. Deuxièmement, on trouve - côté français et italien - des articulations partagées à l'anthropologie, notamment française (Leroi-Gourhan) et à certains auteurs comme Simondon.

À côté de ces points communs, les perspectives italienne et française se distinguent par certaines originalités. La philosophie italienne des techniques comporte des orientations fortement spéculatives, qui peuvent impressionner, ainsi qu'un appétit marqué pour l'exégèse philosophique. Ce travail de commentaire n'est cependant pas qu'herméneutique, il a pour but de « faire éclater les textes bien au-delà de leurs frontières socio-historiques pour en libérer la puissance conceptuelle, au service d'un regard résolument tourné vers le présent» (p. 11). On y trouve d'autre part un ancrage dans la culture humaniste. La philosophie française des techniques se caractérise quant à elle par une attention aux temporalités des techniques et à leur histoire, par un intérêt pour les questions éthiques qui ne relèvent pas de l'éthique appliquée mais qui entrelacent les dimensions ontologiques, anthropologiques et éthiques. Enfin, l'approche française fait de la technoscience une véritable catégorie philosophique.

French Philosophy of Technology et Perspectives italiennes contemporaines en philosophie des techniques comprennent des textes qui donnent à voir des 
angles d'approche originaux sur des objets techniques concrets, couplés à un travail philosophique sérieux d'histoire des idées, de créativité conceptuelle profonde, éclairante et même euphorisante. Mais c'est avant tout le geste éditorial que j'aimerais souligner ici. Ce geste éditorial qui consiste à faire exister - de manière visible - une communauté de philosophes des techniques. J'aimerais à présent me faire l'alliée de cet acte instituant et le prendre au sérieux, en entrant dans les entrailles de ces réseaux d'auteurs pour enquêter sur leurs liens et essayer de donner à voir, à mon tour, mais graphiquement cette fois, ces communautés de philosophes des techniques. Pour y arriver, je vais utiliser les ressources de la science des réseaux, en particulier celles qui sont implémentées dans le logiciel libre Gephi, logiciel initialement développé à l'Université de Technologie de Compiègne, en France, où la philosophie des techniques prend parfois la forme d'une recherche technologique, comme le mentionnent sommairement Sacha Loeve, Xavier Guchet et Bernadette Bensaude-Vincent dans une note de bas de page (p. 12).

À l'aide de Gephi, j'ai donc produit trois graphes : le premier propose une visualisation du réseau des philosophes italiens des techniques qui apparaissent dans le livre Perspectives italiennes contemporaines en philosophie des techniques (Fig. XXI, cahier couleur) ; le deuxième, une représentation du réseau des philosophes français des techniques qui ont été publiés dans French Philosophy of Technology (Fig. XXII, cahier couleur) ; et le troisième, une projection du réseau des philosophes italiens et français de ces deux livres (Fig. XXIII, cahier couleur). Si l'on me permet un encart méthodologique nécessaire, pour produire ces graphes, j'ai entré manuellement chaque auteur présent dans l'un et l'autre ouvrage, ainsi que toutes les sources auxquelles il fait référence dans son article. Pour chaque graphe, j'ai utilisé l'algorithme de spatialisation Force Atlas (force de répulsion 10,000 avec ajustement par la taille), j'ai appliqué le filtre de modularité pour détecter les communautés et j'ai choisi de faire dépendre la taille des nœuds de la centralité intermédiaire, afin d'identifier les individus non pas les plus cités et citants, mais ceux qui se trouvent aux intersections des communautés.

Le premier graphe (Fig. XXI, cahier couleur) propose une visualisation du réseau des philosophes italiens des techniques, tels qu'ils apparaissent dans le livre Perspectives italiennes contemporaines en philosophie des techniques. On peut y voir un petit réseau composé de trois clusters, dont 
l'un - multicolore - est plus grand, dense et connecté que les deux autres colorés en vert et en violet. Le petit cluster en vert est celui de Mauro Turrini et celui en violet est centré sur Frederico Neresini. La structure du graphe pousse notre curiosité à nous interroger sur la spécificité de ces deux auteurs, qui ne partagent visiblement pas de références communes avec le grand cluster multicolore. Mauro Turrini et Frederico Neresini sont tous deux présentés comme des sociologues sur leur page institutionnelle respective ${ }^{1}$. Nous pouvons en déduire que leurs bibliographies s'inscrivent plus dans le champ de la sociologie que dans celui de la philosophie. Autre élément intéressant, ces deux auteurs travaillent sur des techniques peu couvertes par la philosophie des techniques italienne telle qu'elle apparaît dans l'ouvrage, à savoir les techniques informatiques : les instruments haut-débit pour Frederico Neresini, les données personnelles de santé pour Mauro Turrini.

Concernant le cluster dominant, multicolore, on y voit apparaître les différents auteurs de l'ouvrage collectif avec leurs réseaux de références en partie partagés. Côté références, c'est surtout Canguilhem qui se distingue par sa centralité dans le réseau, ainsi que Simondon, Heidegger, Kant et Foucault. L'auteur Rocco Ronchi est présent au milieu du réseau, sans doute parce qu'il travaille beaucoup à partir de Canguilhem et Simondon. Du côté des auteurs justement, on remarque immédiatement la centralité d'Emanuele Clarizio, suivi de près par Xavier Guchet - ce qui se comprend aisément puisqu'il s'agit des deux directeurs de l'ouvrage. On remarque toutefois que le réseau est équilibré, multipolarisé puisque Massimo De Carolis et Antonio Lucci présentent également un haut degré de centralité. En résumé, hormis les deux sociologues qui s'intéressent à l'informatique, nous sommes bien face à une communauté de chercheurs qui partagent des objets de recherche communs et des références communes ${ }^{2}$.

Comparons à présent le réseau des auteurs et références de Perspectives italiennes contemporaines en philosophie des techniques avec celui de French Philosophy of Technology (Fig. XXII, cahier couleur). Le réseau des

\footnotetext{
1. https://www.pastis-research.eu/people/federico-neresini-2/?lang=en ${ }^{\text {et }}$ https://www.iea-nantes.fr/ fr/chercheurs/turrini-mauro_377. Liens consultés pour la dernière fois le 2 septembre 2020.

2. Il serait intéressant de réaliser le graphe des auteurs et références du livre Italian Philosophy of Technology paru chez Springer, dans la même collection que French Philosophy of Technology et de le comparer avec le graphe de Perspectives italiennes contemporaines en philosophie des techniques. Il y a fort à parier qu'on y verrait tout autre chose...
} 
philosophes publiés dans French Philosophy of Technology ne comporte quant à lui qu'un seul grand cluster, plus ou moins densément connecté selon les parties. On remarque immédiatement la centralité des directeurs de l'ouvrage, à savoir Sacha Loeve, Xavier Guchet et Bernadette BensaudeVincent. Cette visualisation permet de mettre en évidence le rôle central joué par ces chercheurs dans l'assemblage de la communauté française des philosophes des techniques. Bensaude-Vincent et Loeve, BensaudeVincent toute seule, Guchet ainsi que Simondon sont également des acteurs absolument centraux de ce réseau. Certains appartiennent à la même communauté (vert pomme).

Au-delà du cœur très entrelacé du réseau, ce graphe donne à voir des communautés qui ont leur spécificité, une quasi-autonomie, tout en restant connectées au noyau central. C'est le cas de Mathieu Triclot, qui porte toute une communauté autour de la recherche sur les jeux vidéo. Mais aussi de Sacha Loeve, qui, lorsqu'il écrit seul, constitue le socle d'une communauté autour des nanotechnologies. Ou encore de Stéphane Vial, dont l'originalité - car c'est encore une originalité pour la philosophie française des techniques, tout comme pour l'italienne - consiste à travailler sur le numérique. Le cas de Stéphane Vial est intéressant car il se trouve relativement proche du noyau central, bien qu'il écrive explicitement s'inscrire en rupture avec une partie de la tradition française et qu'il mène désormais sa recherche de l'autre côté de l'Atlantique. En bas du noyau central, on retrouve "l'école de Compiègne " avec Leroi-Gourhan, Lenay, Stiegler, Stewart, Beaubois et Petit. En haut du noyau central, une communauté en orange, comprenant Hottois, Bontems, Chazal, Bachelard, Latour qui peut intriguer bien qu'un appétit pour les sciences puisse éclairer leurs liens. Le réseau des philosophes français publiés dans l'ouvrage collectif comporte enfin des figures fortes, à l'identité marquée (Larrère, Dupuy, Hoquet, Petit et Guillaume, Puech, Triclot, Loeve, etc.), mais qui n'en constituent pas moins une véritable communauté soudée.

Si l'on compare ce graphe à celui des philosophes du livre italien, on sent peut-être, côté français, une communauté qui aurait eu plus de temps pour mûrir, donnant lieu à de beaux équilibres faits d'autonomie et d'attachements. Là où, chez les Italiens, le champ paraît plus neuf, plus en voie de constitution. 
Enfin, il m'a paru intéressant de produire un dernier graphe (Fig. XXIII, cahier couleur), qui regroupe les philosophes italiens et les philosophes français des deux livres, afin de donner à voir leurs entremêlements et d'essayer de comprendre jusqu'où il s'agit de deux communautés distinctes, plus ou moins en dialogue, ou au contraire d'une même communauté qu'on pourrait qualifier de continentale, latine, vitaliste ou tout simplement franco-italienne.

Sur ce graphe, on distingue deux clusters : l'un est gros, dense, multicolore ; l'autre est petit, excentré et constitué d'une seule communauté, celle de Mauro Turrini. Il est intéressant de noter que lorsqu'on analyse le réseau constitué des auteurs et références des deux livres, le cluster de Frederico Neresini n'existe plus, il a été intégré à la grande communauté des philosophes français et italiens, en rejoignant la communauté colorée en rose dont Bernadette Bensaude-Vincent est le nœud central et en se rapprochant de la communauté de Sacha Loeve. Sans doute que l'ouverture aux STS de ces deux chercheurs et d'une certaine frange de la philosophie française des techniques (plus minoritaire en Italie ?) pourrait expliquer en partie ce phénomène. Pour l'expliciter au mieux, il faudrait toutefois compléter l'approche structurelle et quantitative des graphes par une approche qualitative, en réalisant des entretiens avec les auteurs, qui nous apprendraient certainement l'existence de collaborations, de programmes de recherche menés en commun, etc.

Les communautés constituées par les différents auteurs italiens ont trouvé leur place en se distribuant en divers endroits du réseau global, modifiant au passage sa structure initiale. Avec l'arrivée des philosophes italiens, John Stewart, Vincent Bontens, Simondon, Xavier Guchet, Leroi-Gourhan et Bernard Stiegler se sont par exemple rapprochés, jusqu’à appartenir à la même communauté (vert clair), ce qui n'était pas le cas du réseau franco-français. Mais ce qui saute surtout aux yeux sur cette visualisation, c'est la centralité de Sacha Loeve, Xavier Guchet et Bernadette Bensaude-Vincent, qu'ils écrivent seuls, à deux ou à trois. La constitution de la communauté française et italienne de philosophie des techniques repose donc très largement sur leur travail. Ainsi que sur celui de Simondon (l'importance de ce nœud doit être relevée, et ce d'autant plus que Simondon n'étant pas un auteur du volume, il ne cite personne, sa centralité ne dépend donc que des citations qu'il reçoit, de la part de différentes sous-communautés). 
Les quelques commentaires que je propose ici de ces trois graphes sont loin d'épuiser tout leur potentiel, tout comme les graphes eux-mêmes sont loin d'exprimer tout l'intérêt des deux livres qui nous importent ici. L'ambition de ces graphes est plus modeste. Le geste éditorial de Guchet et Clarizio ainsi que de Loeve, Guchet et Bensaude-Vincent est important en ceci qu'il fait voir le jour à une certaine communauté de chercheurs en philosophie des techniques. Les graphes soumis ici permettent d'aller à la rencontre de ces communautés pour en éclairer les propriétés réticulaires particulières, des propriétés qu'il ne s'agit nullement de généraliser au-delà de ces livres. Ces graphes ne sont toutefois pas autosuffisants et ne possèdent aucun pouvoir explicatif. Ils ne sont intéressants que lorsqu'ils sont discutés, critiqués, réinterprétés, lorsqu’ils invitent les acteurs à préciser certaines positions et appellent le commentaire, lorsqu'ils créent de la surprise, des étonnements, des désaccords, des adhésions. Ce sont des objets qui doivent servir de support aux discussions, qui doivent nous donner envie d'enquêter, de raconter un vécu. Ce sont des objets qui appellent le travail qualitatif de l'enquête sociologique, du terrain, de l'entretien. Un travail que je n'ai malheureusement pas mené à bien ici, dans cette modeste note de lecture.

En guise de synthèse, nous pourrions retenir quelques saillances des graphes de ces deux livres. Premièrement, la communauté française semble avoir bénéficié de plus de temps pour " mûrir " que la communauté italienne. Elle est en effet constituée d'un seul cluster (contre trois en Italie), assez dense, comprenant des nœuds qui flirtent avec les marges tout en restant attachés au cœur du réseau. C'est un réseau robuste et relativement bien distribué, fortement connecté. Deuxièmement, la communauté italienne comporte deux clusters très marginaux, ceux qui touchent à la sociologie et à l'informatique. Troisièmement, lorsqu'on réalise un graphe à partir des données des deux livres (communautés italienne et française, donc), on se retrouve finalement avec un grand cluster et un petit cluster marginal. Le grand cluster montre à quel point les philosophes italiens et français des techniques qui apparaissent dans ces deux livres appartiennent à un même réseau relativement dense, qui tient en grande partie sur la centralité des nœuds constitués par Sacha Loeve, Xavier Guchet et Bernadette Bensaude-Vincent. L'enquête qualitative sous forme d'entretiens nous permettrait certainement d'expliquer ce phénomène. Ces trois chercheurs semblent avoir beaucoup ouvré à la promotion, à la constitution d'une 
certaine philosophie des techniques, en France et en Italie. C'est sans doute l'importance de leurs efforts, en France comme en Italie, qui explique la proximité des deux communautés italienne et française de philosophes des techniques, au point où l'on pourrait se demander s'il ne serait pas artificiel de les séparer.

À côté de ces proximités topologiques (liées à la structure du réseau), les points de rencontre des philosophies italienne et française des techniques sont importants. Un goût pour l'histoire et l'histoire de la philosophie, pour la créativité conceptuelle, pour la réalité concrète des objets techniques, pour le travail de terrain. Mais aussi des références partagées, autour de la lignée Bergson, Canguilhem, Simondon, autour de l'anthropologie et de la sociologie (gardons en tête l'intégration, dans la grande communauté franco-italienne, du cluster italien centré sur le sociologue Mauro Turrini).

Tous ces éléments peuvent être vus comme des encouragements à poursuivre le travail de constitution d'une certaine communauté franco-italienne de philosophie des techniques, telle qu'elle est assemblée par Sacha Loeve, Xavier Guchet et Bernadette Bensaude-Vincent. Une communauté qui pourrait, à écouter ce que les graphes peuvent nous apprendre, gagner en cohérence et en densité si elle parvenait à intégrer les chercheurs qui travaillent sur les techniques informatiques. Ces derniers sont non seulement très peu représentés dans ces deux livres, mais en plus, lorsqu'ils sont présents, ils se retrouvent complètement à la marge du réseau, isolés. Ce phénomène serait-il dû à une possible difficulté d'articuler la lignée vitaliste de Bergson, Canguilhem, Simondon - majoritaire comme on l'a $\mathrm{vu}$ - à l'informatique ? Si oui, alors un chantier pourrait s'ouvrir sur la question. D'autres disciplines comme la sociologie ou les sciences de l'information et de la communication travaillent sur les objets numériques, de possibles collaborations pourraient également être mises en chantier. À moins que ce ne soient les liens directs entre informaticiens et philosophes, par exemple à l'occasion de projets de recherche technologique ou de design, qui gagneraient à être valorisés ? Enfin, pour clore ces quelques propositions embryonnaires, j'aimerais finir sur le partage d'un espoir, d'une demande peut-être : celui de voir nos collègues chercheuses elles 
aussi mises en visibilité comme actrices qui comptent dans la philosophie des techniques franco-italienne ${ }^{3}$.

\section{Bibliographie}

Loeve Sacha, Guchet Xavier et Bensaude Vincent Bernadette (dir.), French Philosophy of Technology: Classical Readings and Contemporary Approaches, Dordrecht, Springer, 2018.

Clarizio Emanuele et Guchet Xavier (dir.), Vie, anthropologie, politique : perspectives italiennes contemporaines en philosophie des techniques, Sesto San Giovanni, Éditions Mimésis, 2019.

\section{L'autrice}

Cléo Collomb est docteure en épistémologie et en sciences de l'information et de la communication. Elle est maîtresse de conférences à l'Université Paris-Saclay, IDEST. Spécialiste de la trace numérique, elle s'inscrit dans une démarche de recherche technologique, visant à développer des instruments d'intelligence des données, pour étudier les dynamiques sociales sur le clear, le deep et le dark web.

3. Des vingt-trois chapitres que comprend French Philosophy of Technology seulement quatre sont écrits ou co-écrits par des chercheuses (dont trois par Bernadette Bensaude-Vincent et un par Catherine Larrère). Quant à Perspectives italiennes contemporaines en philosophie des techniques le constat est plus catégorique : la totalité des contributeurs sont des hommes. 